\title{
Récits du moi multilingue chez Yoko Tawada et José F. A. Oliver
}

La matérialité de l'autre langue

Erzählungen des mehrsprachigen Selbst bei Yoko Tawada und José F. A. Oliver:

die Materialität der anderen Sprache

Narratives of the Multilingual Self by Yoko Tawada und José F. A. Oliver: The

Materiality of the Other Tongue

Hélène THIÉRARD

\section{OpenEdition}

\section{Journals}

Édition électronique

URL : http://journals.openedition.org/ceg/3738

DOI : $10.4000 /$ ceg. 3738

ISSN : 2605-8359

\section{Éditeur}

Presses Universitaires de Provence

\section{Édition imprimée}

Date de publication : 15 novembre 2018

Pagination : 123-140

ISBN : 979-10-320-0183-7

ISSN : 0751-4239

Référence électronique

Hélène THIÉRARD, «Récits du moi multilingue chez Yoko Tawada et José F. A. Oliver », Cahiers

d'Études Germaniques [En ligne], 75 | 2018, mis en ligne le 25 avril 2020, consulté le 29 novembre 2020. URL : http://journals.openedition.org/ceg/3738 ; DOI : https://doi.org/10.4000/ceg.3738 


\title{
Récits du moi multilingue chez Yoko Tawada et José F. A. Oliver
}

\author{
La matérialité de l'autre langue
}

\author{
Hélène THIÉRARD \\ Université Sorbonne Nouvelle / CEREG (EA 4223)
}

Yoko Tawada (1960- ) et José F. A. Oliver (1961- ), deux écrivains contemporains d'expression allemande, pratiquent une écriture à la croisée des langues et des genres littéraires. Tawada, qui a quitté le Japon en 1982 pour vivre en Allemagne, écrit en japonais et en allemand. Oliver, né dans la Forêt-Noire de parents andalous venus comme Gastarbeiter au début des années 1960, écrit quant à lui à partir de ses deux langues maternelles, l'espagnol et l'allemand. Tawada et Oliver font tous deux partie de ce qu'il est convenu d'appeler le ‘corpus Chamisso' en référence au prix Adelbert von Chamisso décerné chaque année de 1985 à 2017 à un ou deux écrivains de langue allemande d'origine étrangère ${ }^{1}$. La critique s'est beaucoup penchée sur cette littérature interculturelle de langue allemande ${ }^{2}$ pour mettre en avant la question de l'identité multiple ou hybride qui s'articule sous la plume de ces auteurs et souligne la créativité spécifique que semble donner le rapport exophone à la langue. Au-delà de ces aspects, la poétique translingue ${ }^{3}$ de Tawada et celle d'Oliver méritent à mon sens d'être rapprochées en ce qu'elles reposent sur une pensée du langage similaire. À partir d'une comparaison des récits du moi multilingue chez Tawada et Oliver,

1. Outre Yoko Tawada (1996) et José F. A. Oliver (1997), citons parmi les écrivains les plus connus à avoir reçu ce prix Emine Sevgi Özdamar (1999), Ilija Trojanow (2000), Feridun Zaimoglu (2005) et Terézia Mora (2010).

2. Voir les ouvrages de Carmine Chiellino (dir.), Interkulturelle Literatur in Deutschland. Ein Handbuch, Stuttgart, Metzler, 2000, et de Helmut Schmitz (dir.), Von der nationalen zur internationalen Literatur : transkulturelle deutschsprachige Literatur und Kultur im Zeitalter globaler Migration, Amsterdam/ New York, Rodopi, 2009.

3. Le terme "translingue " doit être compris dans son acception stricte, à savoir non pas seulement comme la présence latente d'une autre langue (ici le japonais ou l'espagnol) dans la langue d'écriture (l'allemand), mais bien sa présence effective qui donne lieu à des phénomènes d'hybridation langagière. La littérature interculturelle en langue allemande est donc beaucoup plus large que la littérature translingue en langue allemande : voir à ce sujet le chapitre 6 "Fremdschreiben in ZwischenWelten : Zur translingualen Fortschreibung deutschsprachiger Literatur " dans l'ouvrage d'Ottmar Ette, ZwischenWeltenSchreiben : Literaturen ohne festen Wohnsitz, Berlin, Kadmos, 2005, p. 181-203. Ce chapitre porte essentiellement sur Emine Sevgi Özdamar et Yoko Tawada et rapproche leur pratique d'écriture de celle de l'écrivaine chicana Gloria Anzaldúa, figure tutélaire de la création translingue. 
ma contribution propose de mettre en parallèle leur réflexion sur le rôle de la matérialité du langage dans la constitution du sujet. La comparaison pourra sembler déséquilibrée dans la mesure où José Oliver est avant tout un poète et que les récits apparaissent à la marge de son œuvre ${ }^{4}$, alors qu'au contraire dans l'œuvre de Tawada la narration domine ${ }^{5}$. Mais c'est justement de la poésie que je partirai pour analyser les récits du moi multilingue chez Tawada et Oliver, et plus généralement du potentiel de la poésie lorsqu'il s'agit de provoquer chez le lecteur l'expérience de la matérialité de l'autre langue. On saisira ensuite la difficulté de reproduire une telle intensité dans un récit et les enjeux soulevés par cette problématique sur le plan narratif.

\section{Matérialité du langage et constitution du sujet}

Alors qu'on a tendance à oublier la matérialité spécifique de sa propre langue, on est extrêmement sensible à celle de l'autre' langue, et une langue étrangère nous apparait d'abord comme un magma sonore ou une forêt de signes. C'est l'impression suscitée par le poème de Yoko Tawada "MusikMaschineLärm " qui se présente comme de la poésie concrète pour toute personne ne lisant pas le japonais (fig. 1) - or ce poème dédié à Ernst Jandl est publié dans un recueil allemand destiné à un public a priori non japonisant ${ }^{6}$. À l'évidence, la dimension visuelle prévaut ici car elle est coupée de la compréhension du système de signes auquel elle renvoie. Le procédé rappelle celui des Poèmes nègres récités en langue maori ou ewé par Tristan Tzara lors des soirées dada du Cabaret Voltaire en $1917^{7}$. Comme les dadaïstes, Tawada joue sur la curiosité du lecteur : quel sens peut bien avoir en japonais cette suite de signes exclusivement composée de caractères kanji, c'est-à-dire de racines de mots ${ }^{8}$ ? Tout porte à croire que le lecteur japonais est lui aussi face à de la poésie concrète : d'une part, l'absence des caractères hiragana qui transcrivent l'habillage grammatical de la phrase japonaise, d'autre part, la répétition de six kanjis selon un ordre variable. De fait, le contenu sémantique du poème est simple, chaque ligne reproduit les trois termes du titre : " bruit " - 騷音/ sōon; " machine " - 機械/ kikai ; « musique " 音楽/ ongaku. Seul le terme " machine " garde la même position de la première à la dernière ligne; les deux autres, qui ont un kanji en commun (音/ oto - « son »),

4. Oliver a publié une quinzaine de recueils de poésie depuis 1987 , parmi lesquels Auf-Bruch (1987), Duende (1997), fernlautmetz (2000), nachtrandspuren (2002), fahrtenschreiber (2010).

5. Notamment sous la forme de romans comme Das Bad (1989), Das nackte Auge (2004), Schwager in Bordeaux (2008) ou Etüden im Schnee (2014).

6. Yoko Tawada, Abenteuer der deutschen Grammatik, Tübingen, konkursbuch Verlag Claudia Gehrke, 2010, p. 52.

7. Les sources ethnologiques de Tzara sont attestées, cf. Henri Béhar in Tristan Tzara, Euvres complètes, édité et commenté par Henri Béhar, t. I, Paris, Flammarion, 1975, p. 714-718.

8. Dans le même recueil, le poème "Die Mischschrift des Mondes " attire l'attention sur le caractère hybride de l'écriture japonaise qui utilise les idéogrammes chinois pour les racines des mots et les caractères syllabaires hiragana pour "les pieds et les mains " des mots; Tawada, Abenteuer, p. 31. 
MusikMaschineLärm

Nach Ernst Jandl

騒音機械音楽機械騒音機械 音楽機械騒音機械音楽機械 騒音機械音楽機械騒音機械 音楽機械騒音機械音楽機械 騒音機械音楽機械騒音機械 騒音機械音楽機械騒音機械 音楽機械騒音機械音楽機械 騒音機械音楽機械騒音機械 音楽機械騒音機械音楽機械 騒音機械音楽機械騒音機械 騒音機械音楽機械騒音機械 音楽機械騒音機械音楽機械 騒音機械音楽機械騒音機械 音楽機械騒音機械音楽機械 騒音機械音楽機械騒音機械 騒音機械音楽機械騒音機械 音楽機械騒音機械音楽機械 騒音機械音楽機械騒音機械 音楽機械騒音機械音楽機械 騒音機械音楽機械騒音機械

Fig. 1: « MusikMaschineLärm », Nach Ernst Jandl. Yoko Tawada, Abenteuer der deutschen Grammatik, Tübingen, konkursbuch Verlag Claudia Gehrke, 2010, p. 52. 
alternent d'une ligne à l'autre sur une séquence de cinq lignes. Le poème est composé de cette séquence répétée quatre fois à l'identique. En vertu de cette combinatoire, la plupart des signes 音 peuvent être lus comme faisant partie du mot 騒音 ou du mot 音楽 selon le sens de lecture adopté, vertical ou horizontal. Le poème fait ainsi correspondre à une réalité graphique plusieurs réalisations sonores possibles renvoyant à des contenus sémantiques différents. À partir de son expérience de locutrice native du japonais - dont le système d'écriture mixte ne transcrit pas seulement une séquence phonétique sur un support visuel (comme dans les écritures alphabétiques), mais produit aussi des significations sur le plan graphique $^{9}$ (comme le chinois) - Tawada donne une nouvelle impulsion à ce que le dadaïste berlinois Raoul Hausmann initiait en 1918-1920 avec la poésie optophonétique, explorant l'intrication de la matérialité sonore et visuelle du langage. Si cette interrogation est naturellement au cœur de la poésie concrète, il est plus difficile d'imaginer qu'on puisse pousser l'expérimentation aussi loin dans un texte narratif. La réflexion sur les différentes façons de signifier d'une langue à l'autre est certes une constante dans l'œuvre de Tawada et passe souvent, dans ses essais comme dans ses récits ${ }^{10}$, par l'intégration de kanjis dans le texte allemand, mais nulle part ailleurs cette réflexion n'est rendue aussi sensible que dans sa poésie, en raison des possibilités visuelles que celle-ci offre. Nous analyserons en ce sens la dimension sonore et visuelle dans les microrécits du moi des recueils Überseezungen et Talisman ${ }^{11}$, qui rendent compte de l'expérience d'une autre langue par le sujet, et nous verrons en quoi les dispositifs narratifs mis en place vont puiser dans l'expérience poétique.

Dans la poésie translingue de José Oliver, c'est moins la matérialité graphique que la matérialité sonore de l'autre' langue qui est rendue sensible, cela en raison notamment de l'importance du dialecte dans sa constellation multilingue personnelle : l'andalou des parents originaires de Malaga, et l'alémanique parlé à Hausach, le village où il grandit, viennent redoubler le dédoublement des langues maternelles allemande et espagnole. Depuis la fin des années 1980, l'œuvre poétique d'Oliver repose sur l'élaboration d'un dialogue entre ces quatre langues, soit par le biais de l'auto-traduction comme dans le recueil Duende ${ }^{12}$, dans lequel on peut lire chaque poème dans une version allemande, espagnole et alémanique, soit plus généralement par l'hybridation langagière. Les poèmes allemands d'Oliver sont ainsi émaillés de mots et d'expressions espagnols, parfois de vers entiers extraits de la poésie de García Lorca, Jiménez ou Alberti,

9. Sur la conception eurocentrée de l'écriture comme simple transcription de la langue parlée, non constitutive de la signification, on pourra se reporter à Louis-Jean Calvet, Histoire de l'écriture, Paris, Fayard, 2010 [Plon 1996], notamment p. 10-11.

10. On peut ainsi penser au roman de Yoko Tawada, Schwager in Bordeaux, Tübingen, Konkursbuch Verlag Claudia Gehrke, 2008. Traduit par Bernard Banoun, Le Voyage à Bordeaux, Lagrasse, Verdier, 2009.

11. Yoko Tawada, Überseezungen, Tübingen, konkursbuch Verlag Claudia Gehrke, 4e éd., 2013 [2002] et Yoko Tawada, Talisman, Tübingen, konkursbuch Verlag Claudia Gehrke, 8e éd., 2015 [1996].

12. José F. A. Oliver, Duende. Meine Ballade in drei Versionen. Die Ballade vom Duende. La balada del Duende. S Duendenlied, Freiburg, Drey Verlag, 1997. 
et exploitent toutes les possibilités de la typographie et du néologisme pour désautomatiser les mécanismes habituels de production du sens dans la langue ${ }^{13}$. Notre analyse portera sur quelques récits et essais autobiographiques issus des recueils Mein andalusisches Schwarzwalddorf et Fremdenzimmer ${ }^{14}$, dans lesquels Oliver revient sur son bilinguisme (ou quadrilinguisme) précoce et sur le rôle du dialecte dans son devenir poète.

Chez Tawada comme chez Oliver, le récit du moi multilingue est essentiellement le récit de la constitution du sujet 'entre' les langues, dans la différence d'une langue à l'autre. Ces récits d'une expérience de décentrement linguistique qui a lieu dès l'enfance pour Oliver, plus tardivement pour (la narratrice des micro-récits de) Tawada, témoignent d'une prise de conscience existentielle que la relation entre le sujet et le monde est (aussi) une expérience de langage et qu'elle varie d'une langue à l'autre. On est donc très proche chez Oliver et Tawada du propos philosophique de Barbara Cassin concernant la productivité des " intraduisibles " sur le plan épistémologique et politique ${ }^{15}$; or ce qui ne passe pas dans l'autre langue, l'intraduisible 'corps des langues' se compose en grande partie de leur individualité graphique et sonore. Cassin s'appuie elle-même sur une conception du langage de tradition humboldtienne, selon laquelle la diversité des langues est une diversité des Weltansichten : chaque langue est " un "filet irisé" capable, selon son maillage, l'endroit où on le jette, la façon dont on le relève, d'attraper d'autres poissons ${ }^{16}$ ".

La question qui sous-tend ma contribution n'est donc pas tant celle de l'hybridité du sujet mise en évidence dans les récits de José Oliver et Yoko Tawada, mais bien plutôt la question du caractère éminemment physique du processus de constitution du sujet - de tout sujet - dans son rapport au monde à travers le langage. Il ne s'agit pas seulement pour Tawada et Oliver de raconter et d'expliquer comment se constitue une subjectivité dans l'entre-deux des langues, mais aussi de faire prendre conscience au lecteur que sa propre subjectivité s'élabore dans la matérialité du langage, en lui faisant faire lui-même cette expérience dans l'acte de lecture. Nous allons voir que chez Tawada comme chez Oliver, c'est grâce à la dimension matérielle de l'autre langue, tant graphique que sonore, qu'on passe du récit de l'expérience personnelle à un dispositif narratif qui provoque l'expérience du lecteur.

13. Pour une analyse de ces procédés dans la poésie translingue d'Oliver, on se reportera à l'article d'Ana Ruiz, "Wie verhält sich eine interkulturelle Sprache? Eine Fallstudie am Beispiel der Werke José F. A. Olivers ", in Carmine Chiellino, Natalia Shchyhlevska (dir.), Bewegte Sprache. Vom 'Gastarbeiterdeutsch' zum interkulturellen Schreiben, Dresden, Thelem Universitätsverlag, 2014, p. 54-87.

14. José F. A. Oliver, Mein andalusisches Schwarzwalddorf, Frankfurt a. M., Suhrkamp, 2007 ; et id., Fremdenzimmer, Frankfurt a. M., Weissbooks, 2015.

15. Les intraduisibles constituent chez Cassin une méthode de pensée : il s'agit de termes qu'il faut sans cesse retraduire et qui indiquent par là même un nœud conceptuel, cf. Barbara Cassin (dir.), Vocabulaire européen des philosophies. Dictionnaire des intraduisibles, Paris, Seuil-Le-Robert, 2004.

16. Barbara Cassin, Éloge de la traduction. Compliquer l'universel, Paris, Fayard, 2016, p. 50. 


\section{José F. A. Oliver}

La forêt est une image récurrente dans la poésie de José Oliver, où elle se conjugue souvent à celle de la mer, or c'est justement dans la superposition spatiale du "Village andalou en Forêt noire " qu'Oliver dit se sentir " chez lui " (behaust) :

Ich hingegen fühle mich einfach nur behaust und uferkämmend in diesem grünen Meer, das nach Wald und Dämmerfeuchte riecht. Nach Luft, die luftschmeckt, und nach Gedanken, die Gefühle münden; die zur Besinnung kommend, weiterreisen und ein MEHR sich ergründen, an Identitäten. Beileibe nicht Verlust ${ }^{17}$.

Oliver insiste sur le fait que sa double appartenance culturelle et linguistique n'est pas sous le signe de la déchirure nostalgique, mais constitue au contraire une véritable chance, une richesse. La construction syntaxique de ce passage montre assez que l'espace symboliquement superposé mer / forêt (Andalousie / Forêt-Noire) ne forme pas une totalité fermée, mais un dédoublement qui ouvre sur un pluriel (ein MEHR) et initie un mouvement sans cesse relancé. Le sujet est chez lui dans un lieu qui l'incite à rester mobile ; ce voyage continué garantissant l'adéquation des domaines de la sensualité, de la pensée et des sentiments.

Pour comprendre sur quelle réalité repose cette perception d'une Heimat double et une à la fois, il faut rappeler le contexte historique : dans les années 1960, à Hausach, on compte environ trente familles de travailleurs immigrés originaires de Malaga, comme les parents d'Oliver, sans compter les communes avoisinantes. Bon nombre de familles italiennes élisent aussi domicile dans la région et l'existence de cette communauté méditerranéenne modifie le lieu. La Forêt-Noire est donc chez Oliver une région placée sous le signe de l'accueil qui lui a permis de réconcilier ses deux identités ${ }^{18}$, de sorte qu'il peut, une fois adulte, se décrire comme " ne faisant qu'un avec soi dans la contradiction ${ }^{19}$ ". Mais il n'en a pas toujours été ainsi et les récits de soi d'Oliver racontent les tiraillements de l'enfant entre ses deux espaces culturels d'appartenance, l'allemand et l'espagnol. Nous allons voir que le dialecte alémanique joue un rôle déterminant dans l'élaboration d'un sentiment d'identité vécu comme un ancrage physique de la parole dans le lieu : devenu poète, Oliver élabore l'image de celui qui ne fait qu'un " avec un pays qui ne cesse de lui donner naissance et parole à la fois ${ }^{20}$ ". Nous reviendrons plus bas sur l'exploitation poétique de la proximité entre Ort et Wort chez Oliver.

17. José F. A. Oliver, " Mein Hausach ", in Oliver, Schwarzwalddorf, p. 9-15, ici p. 10-11.

18. Sur cette question, nous renvoyons à María Eugenia de La Torre, " Ein Gastling im Fremdenzimmer. Zum Werk von José F. A. Oliver ", in Études Germaniques 287, 2017/ 3, p. 367-382 ; l'article porte sur le multilinguisme, les traditions littéraires et les espaces culturels comme constitutifs de l'identité chez Oliver et s'appuie sur les essais publiés dans les recueils Fremdenzimmer (2015) et Wenn ich einst tot... 1. Entwurf. 1. Tristolog (2016).

19. " eins mit mir im Widerspruch ", ibid., p. 10.

20. " eins mit einer Gegend, die ihn immer wieder wortgebiert », ibid., p. 15. 


\title{
Moi et I'autre
}

Les récits autobiographiques de Oliver parcourent cette enfance andaloalémanique et retracent ce processus de réconciliation entre 'moi' et 'l'autre'. Ces termes n'opposent pas un moi espagnol et un moi allemand, mais un moi perçu comme étant comme les autres et un moi perçu comme différent des autres - or ces positions alternent selon les situations, selon qu'il se trouve l'été chez ses grands-parents en Andalousie ou le reste de l'année à l'école à Hausach.

\begin{abstract}
Augenblicke einer Kindheit, in der ich war wie jeder andere auch. Ein Kenner der heimischen Spiele

und doch stahl sich aus jenen unscheinbaren Idyllen noch jemand mit seiner nicht zu verleugnenden Gegenwart hinaus. Ungetrübt neugierig, bisweilen verletzt. Jemand, der nicht dazugehörte ${ }^{21}$.
\end{abstract}

Si en général l'enfant se sent chez lui dans les deux contextes culturels, les occasions ne manquent pas de sentir à l'intérieur de soi la présence de cet autre comme un Riigschmeckter (ein Fremdling). Or, le refuge que l'enfant se construit très tôt se situe entre les langues, dans l'écriture, et c'est une recherche à laquelle participent ensemble " moi et l'autre " :

Wir waren beide auf der Suche nach Sprache

ich und der Andere ${ }^{22}$.

Oliver a dans cette quête une alliée, Emma Viktoria, la veuve sans enfants chez qui la famille emménage à son arrivée en 1960, et à qui il rend hommage dans le récit intitulé "Zwei Mütter"23 ". Deux mères : " la biologique " et "l'autre ", " la femme qui devait [le] former ${ }^{24}$ ", lui et ses frères et sœur. C'est grâce à cette autre mère que ces enfants de travailleurs immigrés pourront se sentir chez eux de plein droit à Hausach, car dans la rue avec les autres enfants le combat pour l'intégration a lieu en alémanique. Emma Viktoria s'occupe des enfants quand les parents travaillent, elle leur transmet les contes et les chansons populaires, fait faire la prière du matin, prépare la Linzertorte, surveille les devoirs, les emmène ramasser des myrtilles dans les bois - tout cela en dialecte. Oliver évoque son bilinguisme précoce à travers l'image de la maison à deux étages :

Ich bin in einem Haus aufgewachsen, das zwei Stockwerke hatte. Im ersten Stock wurde alemannisch gesprochen, also annähernd deutsch, und im zweiten andalusisch, also annähernd spanisch $^{25}$.

L'enfant est ainsi habitué à parcourir les escaliers entre ces deux étages, d'une mère à l'autre, d'une langue à l'autre. Dans ses récits autobiographiques,

21. José F. A. Oliver, "Wortaus, Wortein ", in Oliver, Schwarzwalddorf, p. 17-30, ici p. 22.

22. Ibid., p. 27.

23. José F. A. Oliver, "Zwei Mütter ", in Oliver, Fremdenzimmer, p. 9-25.

24. Ibid., p. 9-10 : "die leibliche "/ "die andere "; et p. 11 : "die Frau, die uns prägen sollte ".

25. Ibid., p. 16. 
Oliver emploie souvent des expressions dialectales ou des phrases entières rapportées en dialecte alémanique pour évoquer ses souvenirs d'enfance. Dans Fremdenzimmer, celles-ci apparaissent en italique dans le texte avec les explications nécessaires en notes, car le public germanophone auquel s'adressent ces récits est largement non dialectisant. Les bas de pages sont donc bien fournis et le lecteur fait régulièrement le va-et-vient. L'auteur adopte un protocole différent dans le recueil Mein andalusisches Schwarzwalddorf : s'il n'y a pas de notes en bas de page, on trouve en fin de volume un glossaire d'environ cent trente entrées renvoyant d'une part au vocabulaire alémanique et espagnol employé au fil du texte en italique, d'autre part à des éléments culturels et à des traditions régionales.

\section{Matérialité sonore du dialecte : l'expérience du lecteur}

Les notes de bas de page de Fremdenzimmer vont cependant bien au-delà d'une simple aide à la compréhension des termes alémaniques; elles prennent le lecteur à partie et attirent son attention sur la musicalité du dialecte, sur sa matérialité sonore. Ainsi, dans l'épisode de la cueillette des myrtilles, le lecteur non dialectisant bute d'abord sur les paroles rapportées d'Emma Viktoria - " Morge Nochmittag gemmer in d Heibere. Saisch aber de Mama, sie sott eich e alde Hos ruslege, wenn se hit Obed hoimkunnt ${ }^{26}$ " - et cherche automatiquement la traduction au bas de la page. Mais la note ne donne pas la traduction d'emblée, elle incite d'abord le lecteur à revenir aux paroles prononcées et à les lire à haute voix jusqu'à ce que le dialecte sonne et devienne par là même compréhensible :

\footnotetext{
Lesen Sie sich diese Sätze laut vor, dann rückt Ihnen s Alemannische badischer Couleur wahrscheinlich ganz von selbst auf Ihren hochsprachlichen Leib. Für alle, die diesem Ratschlag in die Mundart jedoch nicht folgen wollen - Heibere sind Heidelbeeren : "Morgen Nachmittag gehen wir in die Heidelbeeren. Sag deiner Mutter, wenn sie heute Abend nach Hause kommt, sie möge euch eine alte Hose bereitlegen. " ${ }^{27}$
}

Oliver entend ainsi entraîner son lecteur à parcourir les escaliers de sa maison allemande à deux étages, entre la Hochsprache et une Mundart oubliée mais pas si lointaine, et qui engage le corps tout entier.

Deux textes dans Fremdenzimmer portent plus spécifiquement sur le rôle du dialecte dans l'enfance de l'auteur. Le texte intitulé "Schimpf und Widerstand. Als die alemannische Sprache in mein Schreiben $\mathrm{kam}^{28}$ " raconte le conflit de l'adolescent avec son professeur d'allemand, qui interdit strictement l'emploi du dialecte et corrige la prononciation de ses élèves du haut de sa "Hochsprache " et de sa " höhere Bildung ${ }^{29}$ ". L'intransigeance du " Goethéen ${ }^{30}$ " qui veut l'obliger à renier publiquement son dialecte blesse profondément l'élève de quinze ans qui a

\footnotetext{
26. Ibid., p. 15.

27. Ibid., p. 15 (note 1).

28. José F. A. Oliver, " Schimpf und Widerstand ", in Oliver, Fremdenzimmer, p. 26-34.

29. Ibid., p. 28-29.

30. Ibid., p. 31 et 32 : "Goetheanus " ou " Goetheaner ".
} 
durement bataillé pour son intégration linguistique et a développé un sentiment authentique de soi dans la langue d'accueil que constitue le dialecte. Il perçoit donc l'entreprise de domestication linguistique ${ }^{31}$ de l'institution scolaire comme une remise en cause de sa légitimité :

ich [fühlte] mich ja in jeder Hinsicht unverfälscht und keinesfalls idiomatisch deplatziert. Präziser offenbart: Ich betrachtete mich als echtsprachigen Latsche. Klar wie die Husacher Straßenwirklichkeiten selber - und niemand durfte mir seinerzeit die mühsam errungene Integration ins Hierzulande nachbessern ${ }^{32}$.

Avec le recul des années, Oliver souligne la productivité de ce conflit pour son devenir poétique ultérieur, car si à l'époque l'adolescent se soumet dans le cadre scolaire, il se met aussitôt en devoir d'écrire ses premiers poèmes en langue alémanique sous l'égide de Johann Peter Hebel. Et sans cette étape de "Heimatdichtung pubertaire ${ }^{33}$ ", il ne serait pas devenu le poète translingue qu'il est actuellement, travaillant dans le va-et-vient entre l'allemand, l'alémanique et l'espagnol. Oliver défend moins le dialecte contre la langue écrite que la position mobile qui permet d'aller de l'un à l'autre, l'entre-deux incitant à développer pour son propre usage et dans chaque langue une palette de coloris intermédiaires infinis.

Dans l'essai autobiographique "D Hoimet isch au d Sproch" ", qui porte également sur le dialecte, Oliver puise dans ses souvenirs d'enfance liés à la Fastnacht ou Fasent, l'année où on l'a désigné pour jouer le rôle d'un des rois mages. Le récit de l'expérience d'une vie entre les langues fait ici passer au premier plan l'expérience de lecture entre les langues. Il commence en effet par deux pages intégralement en dialecte - passage en italique assorti d'une note qui là encore encourage le lecteur à lire à voix haute :

Am besten Sie lesen sich den kompletten alemannischen Auftakt dieses Kurz-Essays laut
vor. Dann klingt das Alemannische auch Ihnen ins Ohr. Ich würdige diese eigenwillige
deutsche Sprache unübersetzt, weil es sie so gibt : Besuchen Sie sie! S ist e Erläbnis ${ }^{35}$ !

Contrairement à l'épisode des myrtilles, ces deux pages en dialecte ne sont pas traduites en allemand standard. Le lecteur non dialectisant qui se prête au jeu doit s'engager physiquement dans l'exercice, sa langue accroche, il trébuche sur des sonorités qui lui sont inhabituelles mais retombe sur ses pieds à force de tâtonner. La lecture à haute voix non seulement aide à comprendre l'énoncé en dialecte, mais fait de plus sentir au lecteur qu'il se trouve dans cette zone de 'l'à peu près' évoquée par Oliver : " alemannisch also annähernd deutsch ". C'est justement parce que l'alémanique n'est ni la même langue, ni une autre

31. Le terme de domestication se trouve chez Oliver, qui se compare aux vagabonds " dont on a domestiqué l'identité géographique et qu'on a fait rentrer de force dans le corset de la correction grammaticale " (" die identitätsgeographisch domestiziert und mir nichts dir nichts ins gezähmte Korrektissimo zwangsgrammatikalisiert werden "), ibid.

32. Ibid., p. 26-27.

33. Ibid., p. 32 : "pubertäre Heimatdichtung ".

34. José F. A. Oliver, " D Hoimet isch au d Sproch ", in Oliver, Fremdenzimmer, p. 35-51.

35. Ibid., p. 36. 
langue que le lecteur développe dans cette lecture à haute voix une sensibilité et une sympathie pour la variation langagière qui est une condition de plasticité pour toute langue qui veut rendre authentiquement l'éphémère des sentiments, le vécu, la poésie.

L'avant-propos en dialecte qui introduit le récit de la célébration des rois mages n'a a priori rien à voir avec lui ; il a pour sujet la crise financière et consiste en une série de pirouettes humoristiques à partir du terme Hedgefonds prononcé en dialecte alémanique ("Hätsch-Foh, also hätsch e Fond! "36 "). Ces deux pages ne sont pourtant pas gratuites, et au-delà de la provocation - la crise financière comme la Fastnacht est l'affaire du fou - leur fonction première par rapport à la narration est performative : le lecteur fait l'expérience de la capacité du dialecte à rendre concrets les termes et les raisonnements les plus abstraits. Quoique l'essai s'articule sur le récit autobiographique, il ne porte pas simplement sur le dialecte particulier de l'auteur ${ }^{37}$, mais sur les langues dialectales en général et sur leur potentiel actuel pour développer la plasticité de notre rapport au monde à travers le langage. Oliver fait par exemple observer que l'absence ou quasi-absence du prétérit en alémanique coïncide avec une perception du temps passé différente de celle de l'allemand standard; il met en relation cette expression d'un passé qui continue à influer sur le temps présent avec la force des traditions dans les régions où le dialecte s'est conservé ${ }^{38}$.

Il n'est pas rare qu'Oliver joue sur la typographie pour créer un dialogue entre deux termes proches à une lettre près : W:ort fait entendre $\mathrm{Ort}^{39}$ et souligne l'ancrage de la parole vivante dans le lieu d'où elle émane concrètement. La réflexion sur les variations de perception du monde d'une langue à l'autre, initiée par Oliver dans maints poèmes, essais et récits, repose sur le sentiment d'avoir été dès l'enfance 'lié à la terre' (Erdgebunden $\left.{ }^{40}\right)$ par le dialecte alémanique ; elle se traduit par une redécouverte et une réinvention de ses trois langues d'expression au contact l'une de l'autre. Et si Werden devient sous sa plume W:erden ${ }^{41}$, c'est dans la certitude qu'il n'y a pas de devenir sans ancrage.

\section{YOKO TAWADA}

Les micro-récits de Yoko Tawada prennent souvent la forme d'essais d'ethnolinguistique à la première personne et ils font parfois se rejoindre récit de soi et récit de rêve. Les recueils Talisman (1996) et Überseezungen (2002)

36. Ibid., p. 35.

37. La typographie est très claire à ce sujet : Oliver écrit « m:einen Dialekt », ibid., p. 37.

38. Cf. ibid., p. 41 sq.

39. Par exemple dans le texte " Dichtung und Nachhall ", in Oliver, Schwarzwalddorf, p. 40-64, ici, p. 43 : "Die W:orte hinter dem Wort erzählen Geschichten ».

40. Ibid. À un autre endroit, Oliver oppose sa propre "Landschaftverbundenheit " au manque d'ancrage dialectal du Goethéen qui lui paraît " dialektverwaist ", cf. Oliver, "Schimpf und Widerstand ", p. 28.

41. Oliver, "Dichtung und Nachhall ", p. 63. 
sont composés de ces textes brefs situés au croisement de plusieurs genres ${ }^{42}$. Le récit part en général de situations de la vie quotidienne rapportées par une narratrice à la première personne qui présente de nombreuses ressemblances avec l'auteure. L'apprentissage linguistique et culturel de la jeune femme japonaise sert de base à une réflexion sur les implications culturelles des mots utilisés et sur les découpages spécifiques qu'opère telle ou telle langue dans notre manière de percevoir le monde et notre façon de penser. Cette réflexion repose principalement sur la comparaison entre le japonais langue maternelle et l'allemand langue étrangère, mais intègre également l'expérience d'autres langues étrangères dont Tawada est plus ou moins familière comme l'anglais (" Porträt einer Zunge "), le français ("Musik der Buchstaben ") ou l'afrikaans ("Bioskoop der Nacht " ${ }^{43}$. L'auto-observation est au cœur de ces micro-récits de soi qui confinent parfois à des entrées de journal avec des notes éparses. Si les modes les plus élémentaires de signifier en allemand recèlent une certaine étrangeté pour la narratrice (comme l'existence de substantifs masculins et féminins, de tournures impersonnelles du type " es gibt " ou " es regnet "), dans un second temps le détour par la langue étrangère l'amène à porter un regard étranger sur sa langue maternelle. Or chez Tawada, ce processus de décentrement linguistique et culturel n'est pas seulement d'ordre psychique, il est profondément physique, comme le montre la métaphore des lunettes de chair :

Ich muss mir, um Europa sehen zu können, eine japanische Brille aufsetzen. [...] Diese Brille ist durch meine Augenschmerzen entstanden und wuchs in mein Fleisch hinein, sowie mein Fleisch in die Brille hineinwuchs ${ }^{44}$.

Les récits du moi multilingue qui nous intéressent ici relatent l'expérience d'une métamorphose langagière, c'est-à-dire une modification de la conscience d'être au monde du sujet, de sa subjectivité, vécue au plus profond de son corps. Il serait réducteur d'y voir une position nostalgique déplorant la perte d'un moi unifié et la douleur d'une coupure intérieure. La métamorphose du sujet multilingue ne s'opère certes pas sans douleur, mais nous allons voir qu'elle s'ouvre sur une jouissance liée à la réinvention de soi. La narratrice chez Tawada est mue par le désir d'aller vers d'autres langues comme on va au-devant de sa propre métamorphose, une démarche expérimentale qui a pour but l'ouverture d'un espace de liberté et d'invention.

42. Certains des récits de ces deux recueils ont été traduits en français dans Yoko Tawada, Narrateurs sans âmes, trad. Bernard Banoun, Lagrasse, Verdier, 2001, en particulier "Il ne faut le dire à personne, mais l'Europe n'existe pas ", p. 25-31; "Quelque chose d'étranger sorti de la boîte ", p. 7-11; " Une bouteille vide », p. 57-63; " Musique des lettres », p. 65-67.

43. Yoko Tawada, "Porträt einer Zunge ", in Tawada, Überseezungen, p. 118-152 ; " Bioskoop der Nacht ", ibid., p. 61-91; " Musik der Buchstaben ", ibid., p. 32-35.

44. Yoko Tawada, "Eigentlich darf man es niemandem sagen, aber Europa gibt es nicht ", in Tawada, Talisman, p. 46-52, ici p. 51. 


\section{Métamorphosée en langue (= Zunge), ou la matérialité de l'autre langue (= Sprache)}

Le récit initial de Überseezungen, "Zungentanz ${ }^{45}$ ", fonctionne à la fois comme récit de rêve et récit fantastique, sur un mode qui rappelle $L a$ Métamorphose de Kafka et Le Nez de Gogol. La narratrice raconte un rêve ancien dans lequel " [t]out [s]on corps n'était qu'une langue ${ }^{46}$ ". Suite à cette transformation, étant dans l'impossibilité de trouver du travail, elle décide d'écrire " l'autobiographie d'une langue ${ }^{47}$ " et donne des conférences de ville en ville en Allemagne, ce en quoi il n'est pas difficile de voir une parabole de l'activité d'écrivain de Tawada. La métamorphose n'est pas d'emblée vécue comme traumatique et présente au contraire des avantages sur le plan professionnel, puisqu'elle lui apporte un certain succès d'écrivain. Au-delà de la portée autobiographique du récit, réduire le sujet à la partie de son corps qui est à la fois l'organe de la parole et celui du goût, c'est affirmer que le sujet se constitue avant tout dans le langage, et ce de manière non pas désincarnée mais physique, dans le corps sensible des langues. Or le sujet qui fait l'apprentissage tardif d'une autre langue - comme Tawada et comme la narratrice de la plupart des récits qui nous intéressent - est plus que tout autre sensible à la matérialité de ce rapport au monde dans le langage, hyper-conscient du fait que c'est dans une langue donnée qu'on se représente le monde, et que les modes de signification de cette langue et les associations qui s'y présentent influencent ce qu'on perçoit, conçoit et désire. En ce qu'elle résulte d'une démarche active, la relation sensorielle qui s'instaure entre le sujet et le monde dans l'autre langue diffère de celle qui s'était mise en place de manière inconsciente dans la langue maternelle :

Ich war also ins Japanische hineingeboren worden, wie man in einen Sack hineingeworfen wird. Deshalb wurde diese Sprache für mich meine äußere Haut. Die deutsche Sprache jedoch wurde von mir hinuntergeschluckt, seitdem sitzt sie in meinem Bauch ${ }^{48}$.

On notera l'inversion du rapport contenant/ contenu qui régit ce passage de la langue comme membrane extérieure à la langue ingérée, le sujet se libérant de son moi-peau en devenant moi-ventre ${ }^{49}$. La métamorphose en langue/ Zunge de la narratrice est donc bien à comprendre comme une métaphore des transformations physiques et psychiques par lesquelles passe le sujet en adoptant - en ingérant - une autre langue, un autre rapport au monde ${ }^{50}$.

45. Yoko Tawada, "Zungentanz ", in Tawada, Überseezungen, p. 9-14.

46. Ibid., p. 9 : "Mein Körper bestand aus einer einzigen Zunge. "

47. Ibid., p. 10 : " eine Autobiographie. Die Lebensgeschichte einer Zunge.»

48. Yoko Tawada, "Die Ohrenzeugin ", in Tawada, Überseezungen, p. 95-114, ici p. 103.

49. Sur la fonction contenante du "Moi-peau ", concept développé par le psychanalyste Dider Anzieu à partir de 1974, cf. Didier Anzieu, Le Moi-peau, Paris, Dunod, 1985.

50. À un autre endroit, Tawada rapproche la parole et la perception gustative du réel, qui contrairement à la perception visuelle permettent de dépasser la frontière entre sujet et objet, " car ce qu'on a mangé va dans l'estomac et ce qu'on a dit arrive dans la chair via le cerveau " ("Denn das Gegessene kommt in den Magen hinein und das Gesprochene gelangt durch das 
Mais revenons au fil du récit : ce n'est que dans un second temps que la métamorphose en langue de la narratrice pose problème. Atteinte d'une maladie de la parole qui l'empêche de continuer ses lectures publiques, elle doit consulter un Spracharzt, un " médecin de la parole ${ }^{51}$ " :

Seit einigen Wochen habe ich bei jeder Lesung Schwierigkeiten. Auf dem Manuskriptpapier bilden die Buchstaben eine Mauer, ich gehe geduldig an der Mauer entlang, es gibt aber keine Tür, kein Fenster, nicht mal eine Klingel. Ich kann die Sätze nicht lesen, obwohl ich sie geschrieben habe ${ }^{52}$.

Les phrases sont illisibles pour la jeune femme parce qu'elle ne distingue plus les mots derrière les lettres, d'où l'image des lettres formant un mur. Le problème, tel qu'il est décrit, n'est compréhensible que si l'on considère qu'il s'agit d'une locutrice déjà 'alphabétisée' dans un système d'écriture non-alphabétique, à savoir le japonais, où on lit des mots-idées sans avoir à déchiffrer des lettres au préalable ${ }^{53}$. Le trouble de la lecture témoigne donc d'un dédoublement du moi multilingue : un 'je' est capable d'encoder dans le système d'écriture alphabétique, l'autre 'je', perdu dans ce système d'écriture, est incapable de le décoder.

Lors de la consultation, le médecin lui donne en vain des exercices de prononciation pour reprendre le contrôle de sa bouche, et la langue se met soudain à parler toute seule en japonais " itsudemodonnatokinidemoyomigaettekurusoreyananiwoshitemodoushiyoumonaiarawaittainani ", comme si ses cordes vocales ne lui appartenaient pas, ou plutôt comme s'il s'agissait " de bandes magnétiques dans une machine ${ }^{54}$ ". Nul doute qu'à ce point du récit, la métamorphose de la narratrice en langue rende compte d'une aliénation du sujet victime de son multilinguisme. Le dysfonctionnement physique de la langue (Zunge) et sa maladie disent la perturbation psychique du sujet coincé entre ses deux langues (Sprachen). Cependant, dans l'économie du récit "Zungentanz ", cette phase où sont associés bilinguisme et pathologie mentale n'est que temporaire, prélude (nécessaire?) à la scène finale qui place le bilinguisme sous le signe de la jouissance libératrice ${ }^{55}$.

Gehirn ins Fleisch "), Tawada, " Eigentlich darf man es niemandem sagen ", p. 52; trad. B. Banoun in Tawada, "Il ne faut le dire à personne ", p. 30.

51. On note que les termes "Spracharzt ", "Sprachschmerz " et " Sprachsturz " (sur le modèle de Hörsturz, la perte subite d'audition) sont composés comme si la langue/ Sprache était un organe, cf. Tawada, "Zungentanz ", p. 11.

52. Ibid., p. 10.

53. L'image des phrases sans porte d'entrée ni fenêtre est à rapprocher du propos benjaminien sur la phrase comme " mur " et le mot comme " arcade " dans " La Tâche du traducteur ", texte fondateur sur lequel Tawada revient dans son essai « Das Tor des Übersetzers oder Celan liest Japanisch ", in Tawada, Talisman, p. 125-138, ici p. 135. Voir également Walter Benjamin, Euvres I, Mythe et violence, trad. Maurice de Gandillac, Paris, Denoël, 1971, p. 261-275.

54. Tawada, "Zungentanz ", p. 12 : "Schon wieder springt Japanisch aus meinen Stimmbändern oder sind das fremde Tonbänder in einer Maschine?"

55. Je propose ainsi de nuancer le propos de Yasemin Yildiz quand elle oppose la force libératrice $\mathrm{du}$ bilinguisme chez Tawada à une conception pathologique du bilinguisme chez les tenants du paradigme monolingue. Le trouble psychique lié au changement de langue n'est pas nié dans les récits de Tawada, il en est au contraire constitutif et s'accompagne d'une transformation physique, 
Dans la partie finale du récit en effet, les exercices de prononciation reprennent pour la narratrice-langue, cette fois-ci en rêve et avec son ami Zoltán sous la peau nue duquel elle distingue des veines bleues et rouges comme autant de signes alphabétiques. Alors qu'elle essaie en vain de prononcer des consonnes isolées (les mots Wunsch, Mensch deviennent dans sa bouche "Wunosch", "Menosch ${ }^{56}$ "), son compagnon lui explique comment placer la langue sur le palais pour y parvenir, mais ce jeu logopédique se transforme en jeu sexuel et l'éjaculation magique libère les petites lettres alphabétiques liquides qui ont désormais une fonction toute gustative.

La métamorphose en langue dans "Zungentanz ", qui fait passer la narratrice de la souffrance à la jouissance de l'autre langue, est emblématique de la position de Tawada sur les enjeux du multilinguisme pour le sujet. Nous allons préciser quels sont ces enjeux à partir de quelques récits de soi où le discours prend un tour plus général sur les vertus de l'apprentissage d'une langue étrangère.

\section{Généralisation du récit de soi et expérience du lecteur : se détacher de la langue maternelle}

Comme nous l'avons vu avec la métaphore de la langue-sac, les récits de Tawada mettent à jour une certaine violence de la langue maternelle, d'autant plus insidieuse qu'elle n'est pas reconnue comme telle par le sujet monolingue ${ }^{57}$ :

Ich ekelte mich oft vor Menschen, die fließend ihre Muttersprache sprachen. Sie machten den Eindruck, dass sie nichts anderes denken und spüren konnten als dass, was ihre Sprache ihnen so schnell und bereitwillig anbietet ${ }^{58}$.

Si notre langue tend selon Tawada à réduire nos facultés de pensée et de perception sensorielle, il s'agit moins d'un déterminisme linguistique que d'une paresse due à l'habitude. Contre l'essentialisation du rapport conventionnel entre les mots et les choses, elle préconise le bilinguisme comme entreprise de défamiliarisation linguistique. L’appropriation de la langue étrangère a pour corrélat un détachement salutaire de la langue maternelle et des structures qui influencent inconsciemment notre façon de penser et de percevoir la réalité. L'image de la " dégrafeuse ", souvent citée dans la critique, confère à l'apprentissage d'une langue étrangère un véritable pouvoir libérateur :

ce qui n'empêche pas de désirer intensément le changement de langue pour la libération qu'il apporte. Voir le chapitre sur l'écriture hétérolingue de Yoko Tawada dans Yasemin Yildiz, Beyond the Mother Tongue, The Postmonolingual Condition, New York, Fordham University Press, 2012, p. 109-142, ici p. 118-119.

56. Tawada, "Zungentanz ", p. 13-14.

57. Sur la question du monolinguisme chez Tawada en lien avec Derrida, voir l'article de Dieter Heimböckel, " wie Dreirad und Derrida. Yoko Tawadas Writing back ", in Bernard Banoun, Christine Ivanovic (dir.), Eine Welt der Zeichen. Yoko Tawadas Frankreich als Dritter Raum. Mit dem Tagebuch der bebenden Tage und zwei weiteren Originaltexten von Yoko Tawada, München, Iudicium, 2015, p. 246-263.

58. Yoko Tawada, " Das Fremde aus der Dose ", in Tawada, Talisman, p. 40-45, ici p. 43. 
In der Muttersprache sind die Worte den Menschen angeheftet, so dass man selten spielerische Freude an der Sprache empfinden kann. Dort klammern sich die Gedanken so fest an die Worte, dass weder die ersteren noch die letzteren frei fliegen können. In der Fremdsprache hat man so etwas wie einen Heftklammerentferner : Er entfernt alles, was sich aneinanderheftet und sich festklammert ${ }^{59}$.

On remarquera la portée générale de ce paragraphe où il n'est question ni du japonais, ni de l'allemand, mais du rapport au monde dans le langage qui se construit chez le sujet monolingue. Cette apologie du bilinguisme à des fins de libération du joug monolingue prend parfois une forme sentencieuse : " la langue maternelle fait la personne, alors que la langue étrangère laisse faire la personne ${ }^{60}$ ". Chez Tawada, la libération du signifiant dans la langue étrangère touche parfois jusqu'à la désignation de soi et permet la réinvention du sujet dans l'écriture ${ }^{61}$ : le pronom 'je' désignant la personne qui parle.

Comme le fait justement remarquer Yildiz, le passage de la langue maternelle à la langue seconde ne prémunit pas contre l'essentialisation linguistique, c'est pourquoi les récits de Tawada ne restent pas dans un face-à-face allemand/ japonais ${ }^{62}$ et intègrent l'expérience d'autres langues. Consciente du processus de refamiliarisation en langue seconde, Tawada conçoit l'expérience de la langue étrangère comme un geste critique sans cesse à réitérer ${ }^{63}$. Cette démarche dépasse largement le cadre de ce que l'on a appelé littérature interculturelle de langue allemande $^{64}$ et relève davantage d'une anthropologie poétique du langage. Le récit est une des formes privilégiées, avec l'essai et la poésie, dans laquelle Tawada articule sa pensée du langage. S’il s'ancre dans l'expérience multilingue de leur auteur, c'est avant tout pour que le lecteur prenne part à l'expérience - provisoirement douloureuse - de la défamiliarisation dans l'autre langue.

$\mathrm{Au}$ début de cet article, nous évoquions un poème concret de Tawada qui explore la matérialité visuelle de la langue japonaise, travaillant l'écart possible entre l'image graphique des mots-idées et leur image sonore. Dans d'autres poèmes, comme "Die Mischschrift des Mondes ", Tawada fait faire

59. Yoko Tawada, "Von der Muttersprache zur Sprachmutter ", in Tawada, Talisman, p. 9-15, ici p. 15.

60. Tawada, " Die Ohrenzeugin », p. 111 : " die Muttersprache macht die Person, die Person hingegen kann in der Fremdsprache etwas machen ".

61. Dans le texte " Eine leere Flasche ", la narratrice explique que le " ich " allemand devient son mot favori, un mot parfait pour commencer une histoire parce qu'il est léger et vide de toute information concernant le statut social ou le sexe de la personne désignée. Cf. Yoko Tawada, " Eine leere Flasche ", in Tawada, Überseezungen, p. 53-57.

62. Cf. Yldiz, Beyond the Mother Tongue, p. 141-142.

63. Pour saisir la spécificité de la critique du monolinguisme, souvent au cœur des littératures translingues, par rapport à la critique du langage de la modernité, on pourra se reporter à l'article de Dieter Heimböckel, "Einsprachigkeit - Sprachkritik - Mehrsprachigkeit ", in Till Dembeck, Georg Mein (dir.), Philologie und Mehrsprachigkeit, Heidelberg, Winter, 2014, p. 135-136.

64. Je m'éloigne ici de la lecture de Marion Grein, "Yoko Tawada : Die etwas andere Migrantin. Interkulturalität als literarisch-sprachliche Herausforderung ", in Chiellino, Shchyhlevska (dir.), Bewegte Sprache, p. 139-166. Chez Tawada, la comparaison entre les structures de l'allemand et celle du japonais n'a pas tant pour objectif de réconcilier deux identités culturelles clivées, que de comprendre le rôle des langues dans la constitution de l'être de langage qu'est l'homme. 
au lecteur l'expérience d'un système d'écriture mixte, comme le japonais, en hybridant écriture idéographique et écriture alphabétique. Ses essais, enfin, font saisir ce qui sépare les écritures alphabétiques - qui servent seulement à fixer une séquence phonétique -, des écritures idéographiques qui participent à la conceptualisation sur le plan graphique ${ }^{65}$. Face à la force explicative de l'essai et à la force visuelle de la poésie concrète, on peut se demander quelle serait la force du texte narratif quand il ne s'agit pas moins que de modifier tout le rapport au langage du lecteur occidental, celui-ci ayant intériorisé le principe de la linguistique saussurienne qui affirme la relation de subordination de l'écriture à la langue parlée, et ne prend pour objet d'étude que cette dernière ${ }^{66}$. Tawada nous en fait la démonstration dans le récit à la troisième personne qui met en scène deux amies japonaises, "Die Botin ${ }^{67}$ " : Kayaco, qui doit bientôt partir en voyage pour l'Allemagne, est chargée par Mika d'un important message pour son ancien professeur de musique à Munich. Elle va devoir lui délivrer ce message de vive voix en allemand, langue qu'elle ne parle pas. Le contenu du message étant très sensible - il concerne les raisons de son départ précipité d'Allemagne des années auparavant -, Mika imagine un moyen pour que Kayaco puisse répéter celui-ci en allemand sans pour autant le comprendre : elle lui fait mémoriser une suite d'idéogrammes qui, prononcés en japonais, reproduisent à peu près la séquence phonétique de l'énoncé allemand à transmettre. La " traduction de surface $^{68}$ " sert donc ici à la fois à communiquer (au professeur) et à ne pas communiquer (à Kayaco) le message ${ }^{69}$. Le lecteur allemand se retrouve quant à lui dans une situation similaire à celle de la messagère puisqu'à la fin du récit il découvre le message retraduit en allemand à partir de la suite d'idéogrammes japonais. Cette retraduction de surface - au sens où c'est la surface visuelle des idéogrammes qui est cette fois-ci passée en allemand - donne lieu à un énoncé poétique surréaliste, le passage d'une langue à l'autre et d'une matérialité à l'autre ayant opéré la libération magique du signifiant original.

65. Cf. Tawada, " Das Tor des Übersetzers oder Celan liest Japanisch ".

66. Bernard Banoun revient sur cet aspect fondamental de la démarche de Tawada dans " Notes sur l'oreiller occidental-oriental de Yoko Tawada », Études Germaniques 259, 2010/ 3, p. 415-429, ici p. 419, note 16.

67. Yoko Tawada, " Die Botin ", in Tawada, Überseezungen, p. 44-50.

68. Le terme de " traduction de surface ॥ [Oberflächenübersetzung] est introduit en 1957 par le poète autrichien Ernst Jandl et désigne la traduction du signifiant d'une langue à l'autre par imitation phonétique de l'original. Cette pratique répandue dans la poésie concrète ou oulipienne (Ernst Jandl, Oskar Pastior) est aussi communément appelée traduction homophonique, cf. Till Dembeck, "Oberflächenübersetzung : The Poetics and Cultural Politics of Homophonic Translation ", Critical Multilingualism Studies 3:1, 2015, p. 7-25.

69. Comme le montre Birgit Maier-Katkin, sa conception du langage comme mitteilbar/ unmitteilbar rapproche Tawada de la philosophie du langage de Walter Benjamin, cf. Birgit Maier-Katkin, " Über Polyglotte und Mittelbarkeit. Yoko Tawada im benjaminischen Kontext der Sprache ", Études Germaniques 259, 2010/ 3, p. 455-464. 


\section{L'expérience du lecteur au coeur de la poétique translingue d'Oliver et de Tawada}

Les récits du moi multilingue de Tawada et Oliver présentent l'avantage, par rapport à la poésie concrète, de produire une zone d'inter-subjectivité permettant l'identification du lecteur par le biais de la première personne ${ }^{70}$, qu'il s'agisse d'un "je" autobiographique comme chez Oliver ou d'un "je" (auto)fictionnel comme chez Tawada. Ces récits font ainsi faire au lecteur l'expérience que le sujet se constitue dans la matérialité du langage qui façonne son rapport au monde. Mais l'efficacité spécifique de l'expérience provoquée dans les récits du moi multilingue de Tawada et Oliver vient de ce que les dispositifs narratifs adoptés se nourrissent des dispositifs propres à la poésie concrète et plongent le lecteur dans une expérience sensible de la matérialité sonore et graphique des langues en contact. En ce sens, il n'est donc pas anodin que Tawada et Oliver pratiquent tous deux - dans des proportions différentes - la poésie et le récit.

On voit donc que, si le parcours et l'œuvre de José F. A. Oliver et Yoko Tawada diffèrent à bien des égards, la pensée du langage de tradition humboldtienne qui sous-tend leur poétique translingue présente une grande proximité. Affirmer que la constitution du sujet s'élabore dans la matérialité même du langage, c'est en effet mobiliser à la fois une conception anti-aristotélicienne (non-dualiste) du langage comme diversité des langues et une conception anti-cartésienne (non-dualiste) du sujet comme conscience incorporée, inséparable du corps propre. Cette poétique translingue où la notion d'expérience est essentielle, commune à Oliver et à Tawada dans ses prémisses, produit un rapport au monde complexe et mobile. Cependant la radicalité de Tawada est bien plus grande à cet égard. Les récits-essais autobiographiques d'Oliver, qui apportent un témoignage sur son bilinguisme précoce, retracent le dialogue qu'il a su instaurer au sein de sa double identité culturelle, y compris à travers le travail poétique. Ces récits dépassent la dimension individuelle ou celle d'une minorité biculturelle et accompagnent l'élaboration d'une langue poétique plurielle, certes toujours en mouvement, mais aussi toujours ancrée dans un lieu. Chez Tawada, les récits-essais du moi multilingue puisant dans son expérience personnelle jouent sur les ambiguïtés d'un 'je' mi-autobiographique, mi-fictionnel, et explorent les complexités du sujet de langage dans son rapport au monde. Ils s'inscrivent dans un désir d'errance qui déjoue les fixations et les attributions d'identités en tous genres.

Oliver et Tawada se rejoignent en ce que leur poétique translingue présente une dimension politique forte; leur réflexion sur la subordination du dialecte aux langues nationales pour l'un, de l'écriture à la parole pour l'autre, renvoie l'Europe actuelle à deux idées reçues fermement ancrées dans son histoire culturelle et politique. Les stratégies textuelles visant à provoquer chez le lecteur l'expérience de l'autre langue dans sa matérialité même, que nous avons mises en évidence chez Oliver comme chez Tawada, montrent que tous deux sont

70. Et plus rarement, on l'a vu, de la troisième personne. 
pleinement conscients du rôle actif que les écrivains doivent jouer pour façonner l'Europe de demain. 